A. Pramesh Rao, G. Swarup and Gopal-Krishna, eds.

\title{
GMRT Observations of M 82 and NGC 3079
}

\author{
Judith A. Irwin and D. J. Saikia \\ National Centre for Radio Astrophysics, Pune University Campus, Post \\ Bag 3, Pune, 411007, India
}

\begin{abstract}
We present preliminary GMRT maps of M 82 and NGC 3079 at $327 \mathrm{MHz}$ and $610 \mathrm{MHz}$.
\end{abstract}

\section{Introduction, Observations and Results}

The two bright edge-on spiral galaxies, M 82 and NGC 3079, have provided good test cases for observations with the GMRT during its commissioning phase. This was done primarily at $327 \mathrm{MHz}$, with supplementary $610 \mathrm{MHz}$ data obtained for NGC 3079. The former is the prototypical starburst galaxy and the latter is one of the nearest spiral galaxies harbouring an AGN. Both of these galaxies have nuclear outflows (Seaquist \& Odegard 1991, hereafter SO, Duric et al. 1983).

The galaxies were observed over a variety of dates. For M 82, three $\sim 3$ hour data sets at $327 \mathrm{MHz}$, each with $\sim 6 \mathrm{MHz}$ bandwidth, were combined to form the naturally weighted map shown in Fig. 1, Left. For NGC 3079, the 610 $\mathrm{MHz}$ image (Fig. 1, Right) was made from $\sim 4.5$ hours of observations with a bandwidth of $125 \mathrm{kHz}$.

\section{2. $\quad$ M 82}

We have detected $327 \mathrm{MHz}$ emission from M 82 at the highest spatial resolution yet obtained (for a previous map, see $\mathrm{SO}$ ) including several extensions away from the plane. One source at $\mathrm{RA}=09^{\mathrm{h}} 56^{\mathrm{m}} 15^{\mathrm{s}}, \mathrm{DEC}=69^{\circ} 40^{\prime}$ is a known background source. The data are not sensitive enough to detect the outer halo, as shown in $1420 \mathrm{MHz}$ data of SO, or some of the extended disk emission visible in WENSS data. We expect that these features will show up with supplementary observations.

We have overlaid maps at other wavebands whose resolution is comparable to these observations. Of these, the most interesting comparisons are with the $\mathrm{HI}$ distribution (Yun, Ho, \& Lo, 1993) and the B - I dust distribution (Ichikawa et al. 1994). Within and near the disk, the $\mathrm{HI}$ and radio continuum distributions show some spatial correlation; however, far from the disk, the HI and radio continuum appear to separate. For example, a northerly-directed HI streamer on the east is just offset from a radio continuum extension. Thus, the features appear to be associated with each other, but do not occupy the same volumes. The dust distribution also appears to correlate with the radio continuum. Though not as extensive, the curvature of the contours in the disk as well as the positions and orientations of the dust extensions are similar. Perhaps these features are 

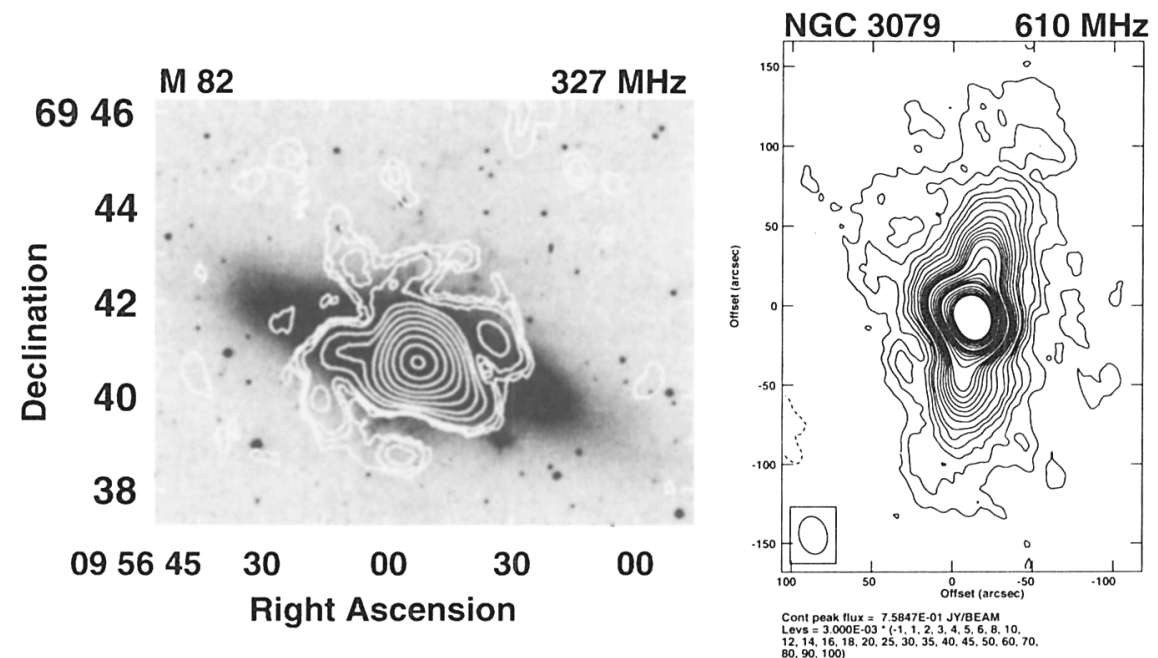

Figure 1. LEFT: $327 \mathrm{MHz}$ map of M 82 over a Digitized Sky Survey image. Contours are at $-6.5,6.5(3 \sigma), 10,16,40,75,160,350,700$, 1500,3000 , and $5000 \mathrm{mJy}$ beam $^{-1}$. The beam is $38^{\prime \prime} \times 26^{\prime \prime} @-11^{\circ}$. RIGHT: $610 \mathrm{MHz}$ image of NGC 3079. The beam is $24^{\prime \prime} \times 18^{\prime \prime}$ @ $13^{\circ}$ and contours are as indicated.

related through the magnetic field. This preliminary finding may provide some insight into the well-known infra-red radio continuum correlation.

\section{NGC 3079}

Both $327 \mathrm{MHz}$ and $610 \mathrm{MHz}$ observations of NGC 3079 show radio emission which extends farther along the minor axis direction than previously shown in Duric et al. (1983). At $327 \mathrm{MHz}$, the $\mathrm{S} / \mathrm{N}$ is low, but a continuum extension can be seen along the western minor axis to a distance of $4.7 \mathrm{kpc}$ and possibly to 9 kpc. $610 \mathrm{MHz}$ maps (e.g. Fig. 1, Right) show emission in the minor axis direction as far as $6.5 \mathrm{kpc}$. There is also a radio continuum loop of similar dimensions to the north-east of the nucleus. This loop shows some spatial correlation with the $\mathrm{H} \alpha / \mathrm{NII}$ image of Heckman, Armus, \& Miley (1990).

\section{References}

Duric N. et al. 1983, ApJ, 273, 11

Heckman T. M., Armus L., Miley, G. K., 1990, ApJS, 74, 833

Ichikawa T. et al. 1994, ApJ, 433, 645

Seaquist E. R., Odegard N., 1991, ApJ, 369, 320

Yun M. S., Ho P. T. P., Lo, K. Y., 1993, ApJ, 411, L17 